\title{
Growth of Mo Large-Scale Fiberform Nanostructures
}

\author{
Shin KAJITA, Tatsuki OKUYAMA ${ }^{1)}$, Hirohiko TANAKA ${ }^{2)}$ and Noriyasu OHNO ${ }^{1)}$ \\ Institute of Materials and Systems for Sustainability, Nagoya University, Nagoya 464-8603, Japan \\ ${ }^{1)}$ Graduate School of Engineering, Nagoya University, Nagoya 464-8603, Japan
}

(Received 13 October 2021 / Accepted 21 October 2021)

\begin{abstract}
We performed helium (He) plasma irradiation to molybdenum (Mo) substrate with auxiliary Mo deposition. Different from W cases, large-scale fiberform nanostructure (LFN) growth did not occur easily. The experiments suggested that the experimental condition of Mo LFN growth has a much narrower conditional window than that of fuzz growth or another hidden control factor exists for LFN growth.
\end{abstract}

(C) 2021 The Japan Society of Plasma Science and Nuclear Fusion Research

Keywords: fuzz, helium plasma, deposition, large-scale fiberform nanostructure

DOI: $10.1585 /$ pfr. 16.1206105

Formation of fiberform nanostructures called fuzz by helium (He) plasma irradiation [1] is an important issue for plasma wall interaction in fusion devices, because it could change the erosion dynamics of material [2,3]. Also, application of fuzz has been explored for optical applications including photocatalysts [4,5]. Recently, an enhanced growth process by additional metal deposition has been found [6]; mm-thick large-scale fiberform nanostructure (LFN) was formed just in an hour of irradiation. Up to now, LFN growth has been identified on tungsten (W), molybdenum (Mo) [6], and rhenium (Re) [7]; however, W substrate was always used for Mo and Re LFN growth. It is noted that the thickness of conventional fuzz cannot reach $10 \mu \mathrm{m}$ even at the fluence greater than $10^{28} \mathrm{~m}^{-2}$ [8]. In this study, based on Mo deposition experiments on Mo substrate, we discuss the mechanism to cause the difference between Mo LFN growth on Mo and W substrates. The influence of the substrate material will shed light on the mechanism of LFN growth. Also, the enhanced growth of Mo is of interest in terms of various applications of its oxides including catalysts, gas sensors, and anode material for lithium-ion batteries [9], as an improvement of the gas sensing performance has been identified on oxidized Mo fuzz [10].

Experiments were performed in the linear plasma device NAGDIS-II. Detail of the experimental setup in the NAGDIS-II device can be found elsewhere [11]. Several $\mathrm{mm}$ away from a $10 \times 10 \mathrm{~mm}^{2}$ substrate, a $0.5 \mathrm{~mm}$ diameter Mo wire (sputtering wire), which is biased to $V_{\text {sw }}<-150 \mathrm{~V}$, is installed upstream side of the substrate. In order to observe the growth process of Mo LFN on Mo substrate, the images are taken by a camera through a viewing port at regular time intervals during the plasma irradiation, as shown in Figs. 1 (a-f). The changes on the substrate started to be observed around $30 \mathrm{~min}$ as forming a white spot at the upper left corner. As time passes, the white area

author'se-mail: kajita.shin@nagoya-u.jp

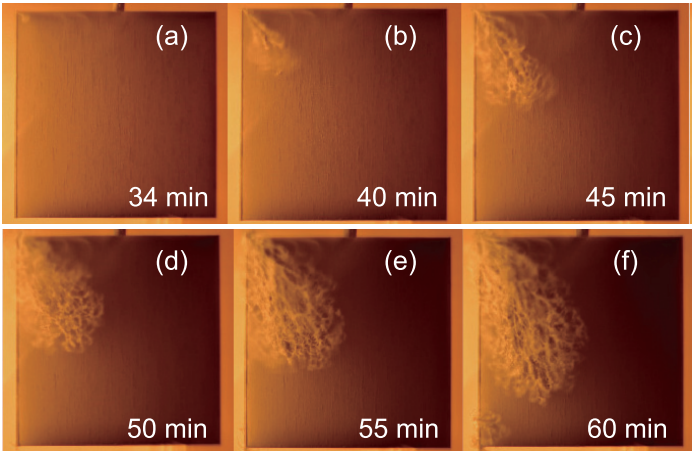

Fig. 1 Pictures of Mo LFN growth on Mo substrate at different irradiation times up to an hour.

spreads in the lower right direction, which is consistent with the growth process of W LFNs [11].

In Fig. 2 (a), red circles and gray squares represent irradiation conditions (the surface temperature $\left(T_{s}\right)$ and $\left.V_{\mathrm{sw}}\right)$ where Mo LFNs were grown and not grown, respectively, on Mo substrate, and blue triangles show the conditions where Mo LFNs were grown on W substrate. Here, the incident ion energy to the Mo substrate is in the range of $60-75 \mathrm{eV}$ for all the cases. Although we tried 17 times in total as changing the irradiation condition, Mo LNFs have been successfully formed only under two conditions. It is noted that $T_{s}$ condition for Mo fuzz growth was known to be $850-1050 \mathrm{~K}[12]$. On W substrate, Mo LFNs were formed higher temperatures $(\approx 1250 \mathrm{~K})$ than the $T_{s}$ window for the fuzz growth. However, no Mo LFN growth occurred when $T_{s}>1050 \mathrm{~K}$ when using Mo substrate.

Figures $2(\mathrm{~b}, \mathrm{c})$ shows picture of the substrate with Mo LFNs shown with red circles in Fig. 2 (a). In Fig. 2 (b) (sample (i)), the growth started from the left top corner and the substrate is black. On the other hand, in Fig. 2 (c) (sample (ii)), the LFNs exists at the lower part of the sample, and the substrate has luster. It is thought that the growth 


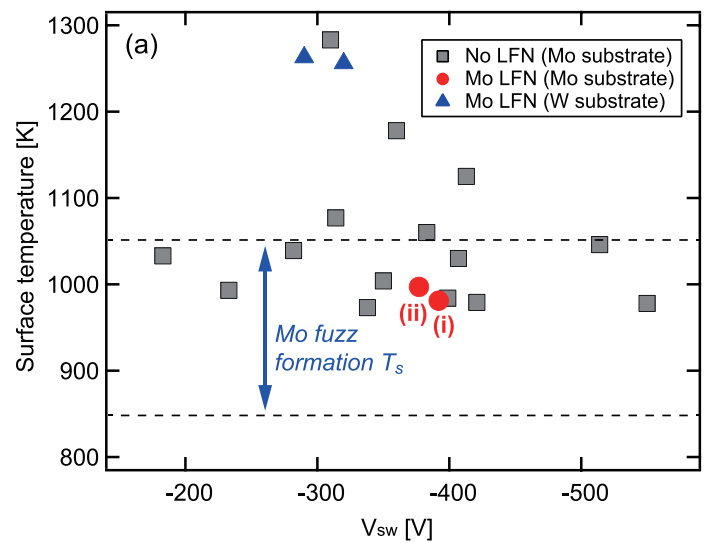

(b)

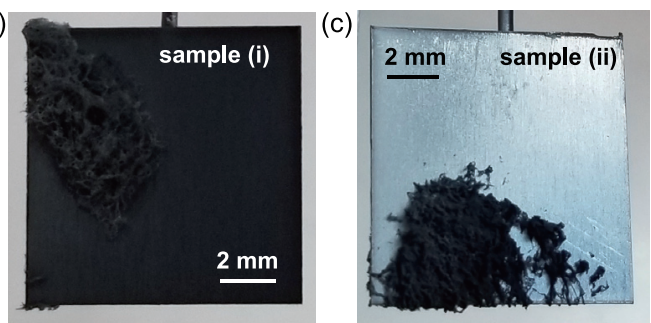

Fig. 2 (a) A summary of the Mo LFN growth at various $T_{s}$ and $V_{\mathrm{sw}}$. Red circles and gray squares represent the cases where Mo LFNs were grown and not grown, respectively, on Mo substrate, and blue triangles show the condition where Mo LFNs were grown on W substrate. (b, c) Pictures of the Mo substrates with Mo LFNs formed at (b) $T_{s}=980 \mathrm{~K}$ and $V_{\mathrm{sw}}=-420 \mathrm{eV}$, and (c) $T_{s}=1000 \mathrm{~K}$ and $V_{\mathrm{sw}}=-420 \mathrm{eV}$.

started from locally formed fuzz at the lower part but could not expanded to upward because of the plasma flow. Figure 3 shows SEM micrographs of the sample (ii). In addition to thick fiberform structures shown in Figs. 3 (a, b), membrane structure is observed, though not shown here, similar to W LFNs [13]. Figure 3 (c) shows the boundary of the LFNs and the substrate, revealing that fuzz was not grown on the substrate. Rather, undulating surface, which was observed on $\mathrm{W}$ surface after He plasma irradiation at lower fluence $\left(<10^{25} \mathrm{~m}^{-2}\right)$ or temperature $(<1000 \mathrm{~K})[14,15]$, can be seen (Fig. $3(\mathrm{~d}))$. The result suggested that Mo deposition counteracted fuzz growth on sample (i) even though the difference in the irradiation condition was subtle.

Figure 2 (a) suggested that the conditional window of the LFN growth is much narrower than that of fuzz growth, as was identified in the temperature window of W LFN growth, whose minimum threshold $T_{s}$ increased by 150 $300 \mathrm{~K}$ from that of fuzz [16]. The shift in the condition may be related to the fuzz growth process under deposition condition, because fuzz growth is thought to be prerequisite for the LFN growth [11]. Enhanced growth of W fuzz by auxiliary deposition has been identified in a magnetron sputtering and Magnum-PSI in addition to $\mathrm{W}$ LFNs $[17,18]$. However, as shown in Fig. 2 (c), the deposition would counteract the fuzz growth under certain con-
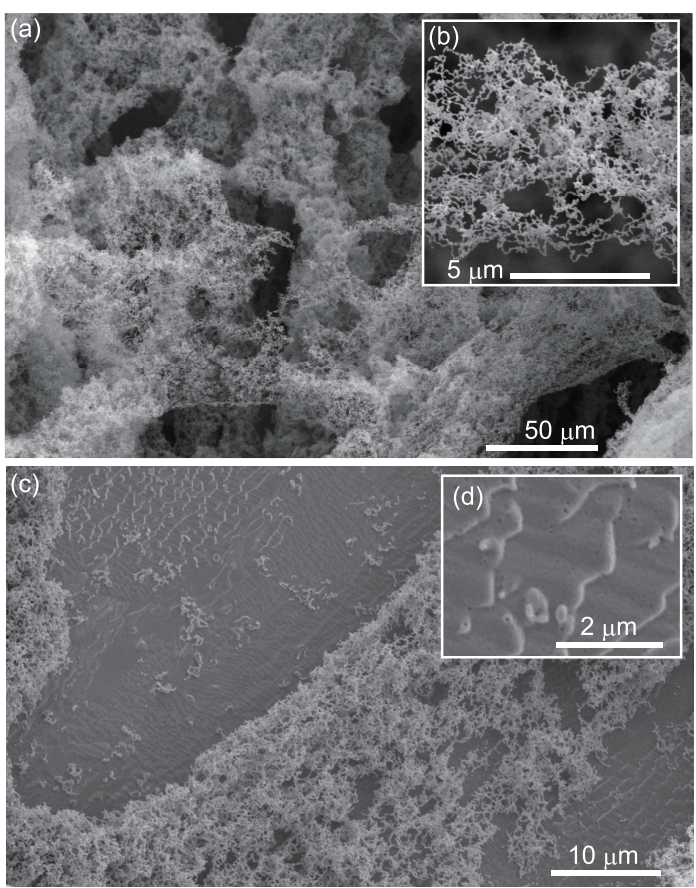

Fig. 3 SEM micrographs of Mo LFNs grown on a Mo substrate (sample (ii)). The irradiation condition is as follows: $T_{s}=1000 \mathrm{~K}$, the He flux of $6.9 \times 10^{21} \mathrm{~m}^{-2} \mathrm{~s}^{-1}$ and $V_{\text {sw }}=-420 \mathrm{~V}$.

dition. Another factor to cause the difficulty in the growth of Mo LFNs on Mo substrate can be the He flux. In this series of experiments, He flux decreases with decreasing $T_{s}$, because the sample temperature is determined mainly from the balance between the heat influx from the plasma and radiation. Thus, we cannot deny the possibility that there is He flux threshold for Mo LFN growth. For future research, it is of importance to perform experiments under the condition where $T_{s}$ can be controlled independently by cooling or heating the sample.

This work was supported in part by a Grant-in-Aid for Scientific Research (B) 19H01874, Grant-in-Aid for Challenging Research 21K18617, and Fund for the Promotion of Joint International Research 17KK0132 from the Japan Society for the Promotion of Science (JSPS).

[1] S. Takamura, N. Ohno, D. Nishijima and S. Kajita, Plasma Fusion Res. 1, 051 (2006).

[2] S. Kajita, G.D. Temmerman, T. Morgan, S. van Eden, T. de Kruif and N. Ohno, Nucl. Fusion 54, 033005 (2014).

[3] G. Sinclair, J.K. Tripathi and A. Hassanein, J. Appl. Phys. 123, 133302 (2018).

[4] M. de Respinis, G. De Temmerman, I. Tanyeli, M.C. van de Sanden, R.P. Doerner, M.J. Baldwin and R. van de Krol, ACS Appl. Mater. Interfaces 5, 7621 (2013).

[5] S. Kajita, K. Miyaguchi, H. Tanaka, E. Yasunaga, T. Yoshida and N. Ohno, J. Photochem. Photobiol. A: Chemistry 418, 113420 (2021).

[6] S. Kajita, S. Kawaguchi, N. Ohno and N. Yoshida, Sci. Rep. 8, 56 (2018). 
[7] S. Kajita, T. Nojima, T. Okuyama, Y. Yamamoto, N. Yoshida and N. Ohno, Acta Materialia 181, 342 (2019).

[8] T. Petty, M. Baldwin, M. Hasan, R. Doerner and J. Bradley, Nucl. Fusion 55, 093033 (2015).

[9] L. Mai, F. Yang, Y. Zhao, X. Xu, L. Xu, B. Hu, Y. Luo and H. Liu, Materials Today 14, 346 (2011).

[10] K. Ibano, Y. Kimura, T. Sugahara, H.T. Lee and Y. Ueda, Jpn. J. Appl. Phys. 57, 040316 (2018).

[11] S. Kajita, S. Kawaguchi, N. Yoshida, N. Ohno and H. Tanaka, Nucl. Fusion 58, 106002 (2018).

[12] S. Takamura, Plasma Fusion Res. 9, 1405131 (2014).

[13] S. Kajita, N. Yoshida, S. Kawaguchi, H. Tanaka, N. Ohno, D. Nagata and M. Tokitani, Nucl. Mater. Energy 18, 339
(2019).

[14] N. Ohno, Y. Hirahata, M. Yamagiwa, S. Kajita, M. Takagi, N. Yoshida, R. Yoshihara, T. Tokunaga and M. Tokitani, J. Nucl. Mater. 438, S879 (2013).

[15] R. Sakamoto, E. Bernard, A. Kreter and N. Yoshida, Nucl. Fusion 57, 016040 (2017).

[16] S. Kajita, N. Yoshida and N. Ohno, Nucl. Mater. Energy 25, 100828 (2020).

[17] P. McCarthy, D. Hwangbo, M. Bilton, S. Kajita and J.W. Bradley, Nucl. Fusion 60, 026012 (2020).

[18] S. Kajita, T. Morgan, H. Tanaka, Y. Hayashi, N. Yoshida, D. Nagata, J. Vernimmen, S. Feng, R. Zhang and N. Ohno, J. Nucl. Mater. 548, 152844 (2021). 